\title{
Editorial
}

\section{New Reviewer Mentoring Program}

Trainees often contact us and ask how they can get more involved in the review process. At the Journal of Neuroscience and eNeuro we are committed to inclusive peer review and to training the next generation of outstanding reviewers. For example, editors at eNeuro have developed materials so that prospective reviewers can observe a fair and rigorous review by experienced reviewers and editors. At JNeurosci, we are proud to announce a new Reviewer Mentoring Program that will provide one-on-one training to help advanced graduate students and early postdocs, or other SfN members interested in learning how to provide constructive and fair reviews from some of our most committed and highly respected reviewers. Note the potential trainee must have already been a 1st author on a published paper to participate.

This program will launch as a pilot on January 16, 2018.

To get started, a Reviewer Trainee will select a potential Reviewer Mentor from the list and contact them through the website. When matched, they will download the training materials from the Trainee Resources page in Neuronline. After reviewing the materials, the Trainee will choose an article in an appropriate area of neuroscience from bioRxiv. Note that articles submitted to eNeuro have the option of uploading a preprint to bioRxiv at the time of submission, so papers under consideration may be chosen for review.

Once the paper is chosen, the Trainee will draft a review and send it to their Mentor. The Mentor will engage constructively with the Trainee to point out strengths and weaknesses of the review to guide in the revision process. We expect that during this Revision Stage, the draft will be sent back and forth for editing until both the Mentor and the Trainee are satisfied with the final version. Trainees will then have the option of posting the review to the preprint on bioRxiv to provide feedback to authors. Trainees will also be added to a database of trained reviewers; strong reviewers may be invited to review future manuscripts.

We think that this program provides a number of services to the neuroscience community. First, it will provide guidance to trainees on what is expected in a fair and constructive manuscript review. Second, it will provide an entry into the review process for advanced trainees who are skillful reviewers. Finally, if reviews are posted to bioRxiv, it will help promote prepublication peer review, and that will ultimately result in more feedback to authors, more openness in review, increased diversity in the reviewer pool, and early access to peer-reviewed data.

We hope that trainees will be interested in taking part in this pilot program, and we welcome feedback on how to enhance the process in the future.

As always, we are eager to hear from you on what we can do better. Please e-mail me at JN_EIC@SFN.org or Tweet to @MarinaP63 with questions and comments.

(D) Marina Picciotto, EiC, JNeurosci

DOI:10.1523/JNEUROSCI.3653-17.2017 